\title{
Recognition of Electromyographic Signal Time Series on Daily Hand Motions Based on Long Short-Term Memory Network
}

\author{
Jin Hua ${ }^{1 *}$, Qinkun Xiao ${ }^{1}$, Li Wang ${ }^{2}$, Yixin Liu ${ }^{1}$, Xuhui Ning ${ }^{1}$ \\ ${ }^{1}$ College of Electronic Information Engineering, Xi'an Technological University, Xi'an 710021, China \\ ${ }^{2}$ The National Research Center for Rehabilitation Technical Aids, Beijing 10076, China
}

Corresponding Author Email: huajin@xatu.edu.cn

https://doi.org/10.18280/ts.380216

Received: 15 November 2020

Accepted: 4 February 2021

\section{Keywords:}

surface electromyographic (sEMG) signals, EMG signal analysis, long short-term memory (LSTM), action recognition

\begin{abstract}
Electromyographic (EMG) signals contain various information about muscle actions, such as intensity and time. In most studies on EMG signals of hand motions, the model is trained on a single action dataset before being applied to action recognition. But rehabilitation training aims to enable the patient to make daily actions, each of which encompasses a series of individual actions. Because EMG signals are noisy and non-stationary, the transition between individual actions could result in a high error in action recognition. This paper designs the common composite actions in daily life, and then obtains the EMG signal data of continuous actions. The signal segments of the composite actions were determined to derive the static and dynamic states of each action. With the aid of the sliding window, the authors obtained the time series data of the eight-channel EMG signals of each composite action, which vary with the elapse of time. Then, the time series data were transformed into the time-frequency image flow of the corresponding composite action. The ten individual actions of the designed composite hand actions were recognized by a self-designed model, which couples three-dimensional convolutional neural network (3D CNN) with long shortterm memory (LSTM) network. The recognition rate was as high as $92 \%$. Finally, an interactive simulation environment was constructed for hand actions on Unity5.3.1f1. Under the environment, the accuracy of controlling the movement of an unmanned vehicle with hand actions was measured. The results show that the recognition rate of our method stabilized at $90 \%$.
\end{abstract}

\section{INTRODUCTION}

Electromyographic (EMG) signals have been widely applied in medical diagnosis, rehabilitation treatment, action recognition, and human-machine interaction (HCI). Surface EMG (sEMG) signals refer to the weak nonlinear and nonstationary signals produced by the physiological changes of muscle fibers, which are the result of the time-domain superposition between the EMG signals from multiple muscle action units covered by sampling electrodes. Carrying a wealth of information on biological activities, sEMG signals have a great research value in the field of medical rehabilitation, sports health, and pattern recognition $[1,2]$.

Beckman Institute for Advanced Science and Technology, a unit of the University of Illinois at Urbana-Champaign, carried out experiments on EMG signals, and observed that the subjects and the manipulator took the same actions in response to the same EMG signal [3]. Gesture recognition experiments have confirmed that human gestures can be identified through EMG signal recognition [4].

Currently, EMG signals are generally acquired by two methods. The indwelling or intramuscular EMG signals are detected with needles or wires inserted into muscles, while the sEMG signals, i.e., the sum of potentials as the muscles contract, are obtained by electrodes pasted on the skin [5-8].

In this paper, the second method is adopted to acquire the sEMG signals. Based on these signals, the authors analyzed the EMG activities of muscles, and determined the functional and morphological changes of neuromuscular system [9-11].

\section{LITERATURE REVIEW}

The key issue of EMG control system is the accurate recognition of user's movement intentions $[12,13]$. As a result, researchers have always been trying to improve the recognition rate of sEMG signals. In fact, how to improve the recognition rate is a research hotspot in the field of sEMG signal recognition.

On the sEMG signal recognition of hand actions, neither time-domain feature analysis nor frequency-domain feature analysis could achieve the desired classification effect [14-16]. Based on time-frequency domain features, however, the feature points can be allocated to clear classes, improving the recognition rate of the classifier. In recent years, the timefrequency domain feature analysis mainly focuses on shorttime Fourier transform (STFT) and wavelet transform [17, 18]. Both of them amplify or reduce the scale of the frequency domain, using the time scale factor [19-22].

To improve the poor resolution of wavelet transform in high-frequency bands, Du et al. [23] put forward a decomposition method called wavelet packets transform (WPT). In addition to low-frequency data, the WPT can decompose high-frequency signals, making up for the defect 
of wavelet transform. Luo and Yan [24] extracted the features of sEMG signals by time-frequency domain method, performed pattern classification of sEMG signals, and realized the control of myoelectric prostheses. To solve the weakness of traditional wavelet thresholding on the denoising of sEMG signals, Ma et al. [25] proposed a denoising method through exponential and logarithmic approximations, and applied this improved thresholding approach to remove the noises from sEMG signals. Based on the amplitude-time feature recognition in electromyogram, Atzori et al. [26] opened a new way to estimate complex body actions, and proved that their method can effectively classify the sport skills of the subjects, according to the energy contribution of muscles to the movement.

EMG signals have both spatial and temporal features. However, convolutional neural network (CNN) can only extract spatial features, failing to mine the time-domain features. During deep learning, the recurrent neural network ( RNN) is usually adopted to handle time domain problems. With a looped architecture, the RNN is equivalent to a neural network whose values are assigned multiple times. It is capable of preserving information in the long term. However, the RNN faces the vanishing gradient problem. To overcome the problem, Hochreiter and Schmidhuber [27] introduced the storage unit to the traditional RNN, creating the long shortterm memory (LSTM) network, which avoids long-term dependence.

\section{DESIGN AND TRAINING OF LSTM MODEL}

\subsection{Design of composite action and preparation of dataset}

The continuous hand actions were collected in the following manner: Each composite action contains ten actions, which are

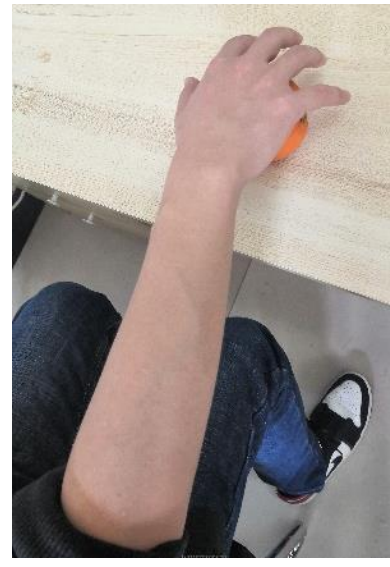

(a) Initial state

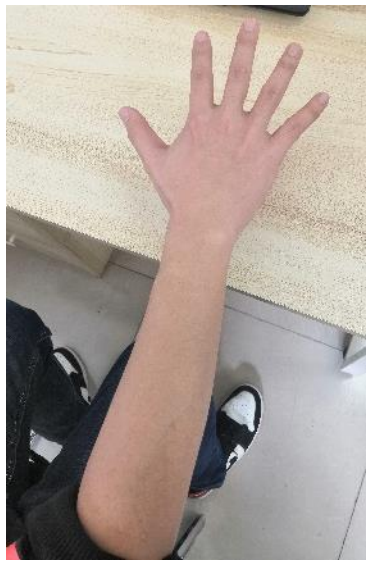

(b) Palm opening

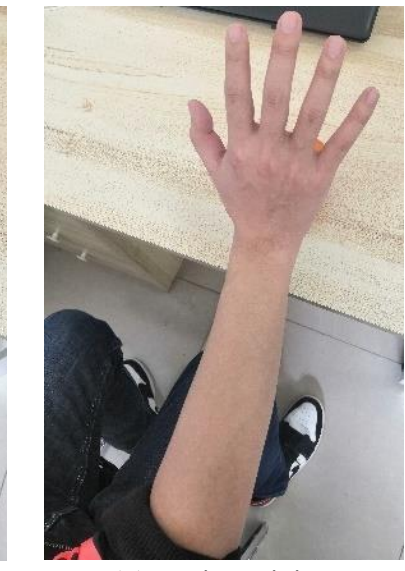

(c) Wrist raising in turn palm opening, gripping, wrist raising, wrist lowering, wrist left bending, wrist right bending, wrist counterclockwise rotation, wrist clockwise rotation, arm raising, and arm lowering. The subjects were asked to maintain each action for $3 \mathrm{~s}$ before starting to take the next action. After completing a composite action, the subjects took a break for $1 \mathrm{~min}$. After the subjects resumed the initial action state, the next composite action would be collected. A total of thirty composite actions were collected from each subject. The collected data were transmitted to a computer, and stored as a composite action dataset. Then, the dataset was divided into three subsets: a training set $(80 \%)$, a test set $(10 \%)$, and a verification set $(10 \%)$.

Recently, neuroscientists have discovered that humans acquire the movement ability through observation and imitation. When a person observes and imitates the others' movement, his/her brain does not perceive a continuous and smooth movement, but a composite action made up of several actions arranged in different sequences. The smallest movement units are referred to as action primitives [28]. This paper decomposes daily actions into countless action primitives. Then, a composite action can be recognized and classified by identifying the sequence and class of its action primitives. The daily composite actions and their action primitives of this research were defined as follows:

The composite action of ball bouncing is completed by the up and down movements of the wrist. It can be broken down into three actions (Figure 1): palm opening, wrist raising, and wrist lowering. EMG signal acquisition began with the initial state. The subject was asked to maintain each action for $3 \mathrm{~s}$ before starting to take the next action. After completing a composite action, the subject relaxed his/her hand for $5 \mathrm{~s}$. Then, he/she would resume the initial state, and got ready for the acquisition of the next composite action. Each composite action of ball bouncing lasts $9 \mathrm{~s}$.

Figure 1. Signal acquisition flow of ball bouncing

The composite action of hand waving is completed by the left and right movements of the wrist. It can be broken down into four actions (Figure 2): palm opening, arm raising, wrist left bending, and wrist right bending. After completing the four actions, the subject had $5 \mathrm{~s}$ to relax his/her palm and withdraw the arm, resuming the initial state. Each composite action of hand waving lasts $12 \mathrm{~s}$.

The composite action of water drinking lasts $18 \mathrm{~s}$, longer than the above two composite actions. It can be broken down into six actions (Figure 3): palm opening, wrist clockwise rotation, gripping, arm raising, arm lowering, and palm opening. After completing a composite action, the subject also relaxed for $5 \mathrm{~s}$, and resumed the initial state.

Following the above signal acquisition flows, each of the three daily composite action was repeated 10 times. Every subject rested for 5-10min between taking different composite actions. The collected data were transmitted to the computer for storage. Besides, the EMG signals were imported to the trained composite action recognition network for recognition. 


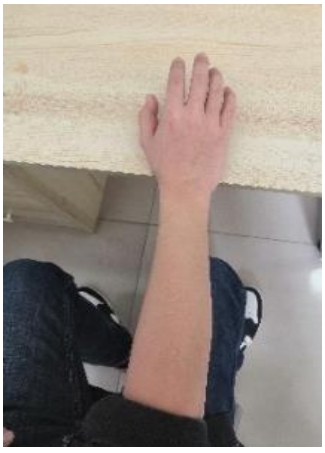

(a) Initial state

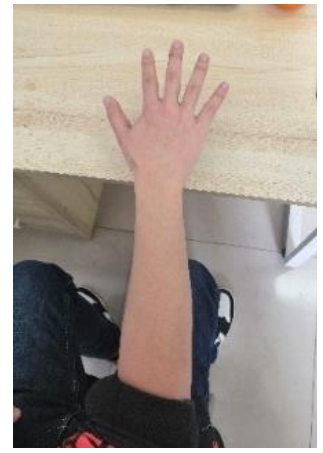

(b) Palm opening

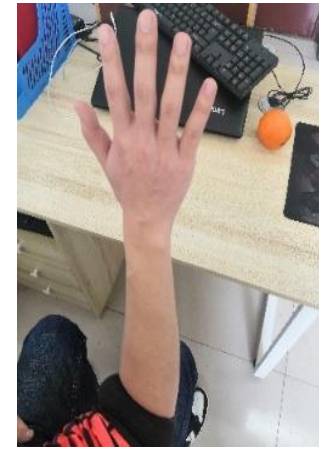

(c) Arm raising

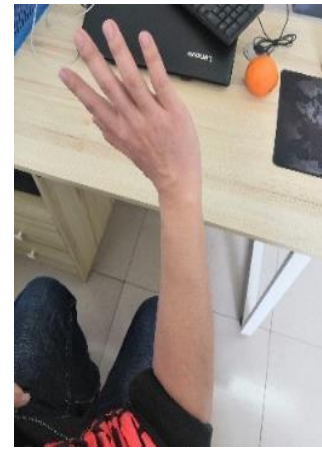

(d) Wrist left bending

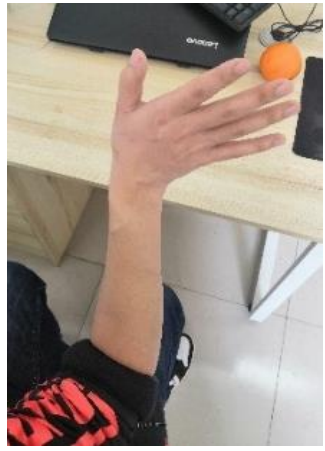

(e) Wrist right bending

Figure 2. Signal acquisition flow of hand waving

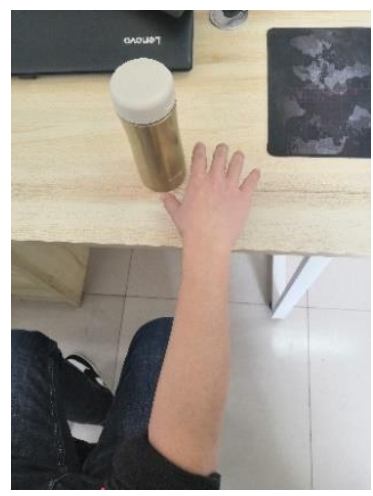

(a) Initial state

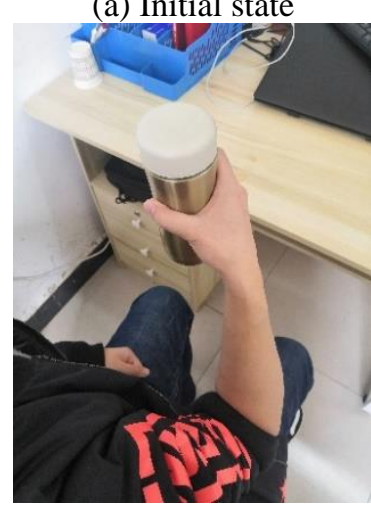

(e) Arm raising

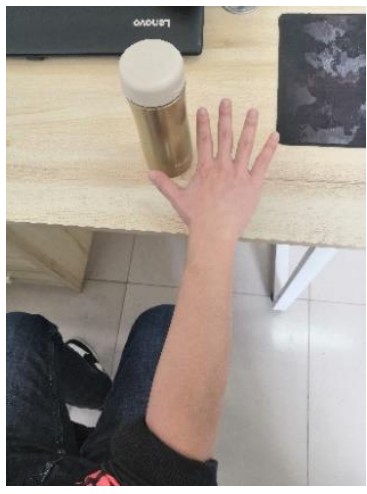

(b) Palm opening

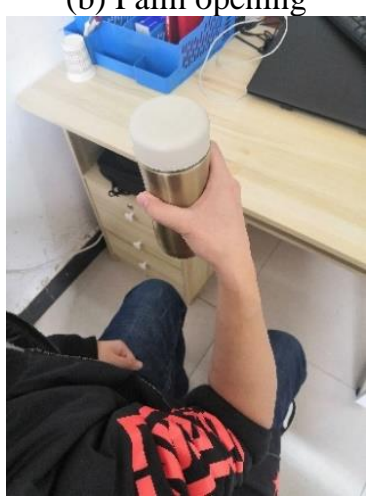

(f) Arm lowering

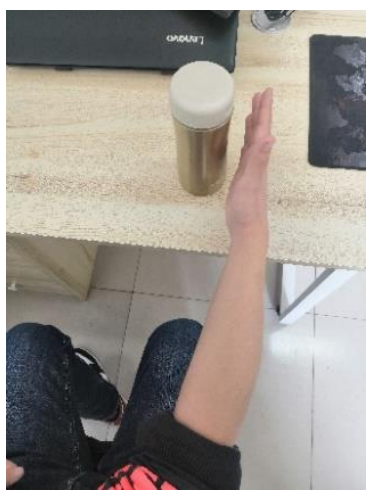

(c) Wrist clockwise rotation

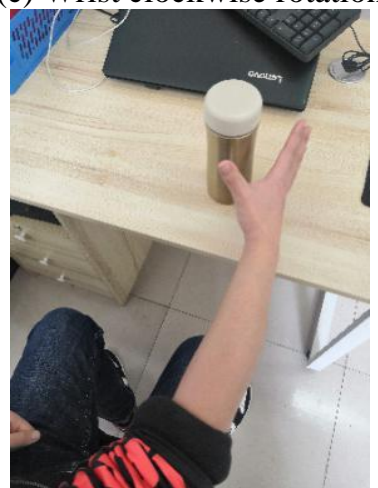

(g) Palm opening

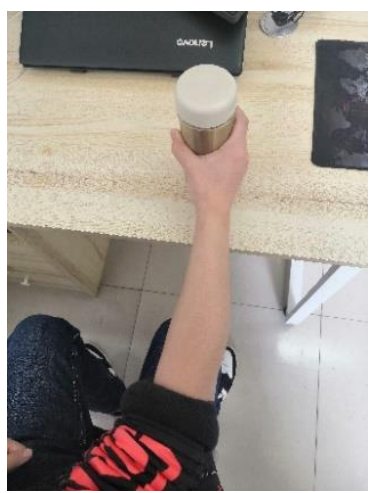

(d) Gripping

Figure 3. Signal acquisition flow of water drinking

\subsection{Modeling of composite action recognition network}

\subsubsection{Composite action recognition model}

Each continuous daily action can be decomposed into isolated action primitives, that is, multiple single actions. Thus, the recognition of a daily action is equivalent to the recognition of each action making up the composite action.

Figure 4 shows the flow chart of the composite action segmentation network.

First, the EMG signals of a composite action are preprocessed. If the signals are currently static, the corresponding action must be relaxation. If the signals are currently dynamic, the recognition network will be called for training and recognition. The network training uses the EMG signals collected from the composite action. The trained recognition networks for single actions will be applied to recognize the composite action. The parameters of the composite action recognition network will be adjusted and optimized to obtain the final weights and biases of the network.

\subsubsection{Transfer learning of action signals}

Figure 5 shows the structure of the action recognition network in time series of EMG signals based on CNN + LSTM (ConvLSTM). To fully mine the features from the timefrequency diagram of EMG signals, the recognition network was formed by the stacking of 3 deep CNN (DCNN) layers, 2 ConvLSTM layers, 1 fully-connected (FC) layer, 1 G-way FC layer, and 1 Softmax layer. Tables 1 and 2 list the parameter settings of the DCNN and ConvLSTM modules, respectively.

The short- and long-term spatiotemporal features were extracted from each channel. The outputted features were spread as a one-dimensional (1D) line for feature fusion. All features were imported to the FC. The number of action classes was set to 10 for the calculation unit in the G-way FC. Finally, the actions were summarized and output by the Softmax layer, clarifying the class of each action.

This paper applies transfer learning to evaluate the recognition effect of the EMG signals of composite actions. Firstly, the action recognition network was trained on every single action dataset. Then, the learned features were used to 
train the network on each composite action dataset, which helps to shorten the training process and prevent overfitting. Through transfer learning, the training speed was accelerated, the model converged earlier, and the recognition rate was improved. The network parameters were randomly initialized, and finetuned by Bayesian optimization. The output size was set to 10 , and the learning rate to 0.001 .

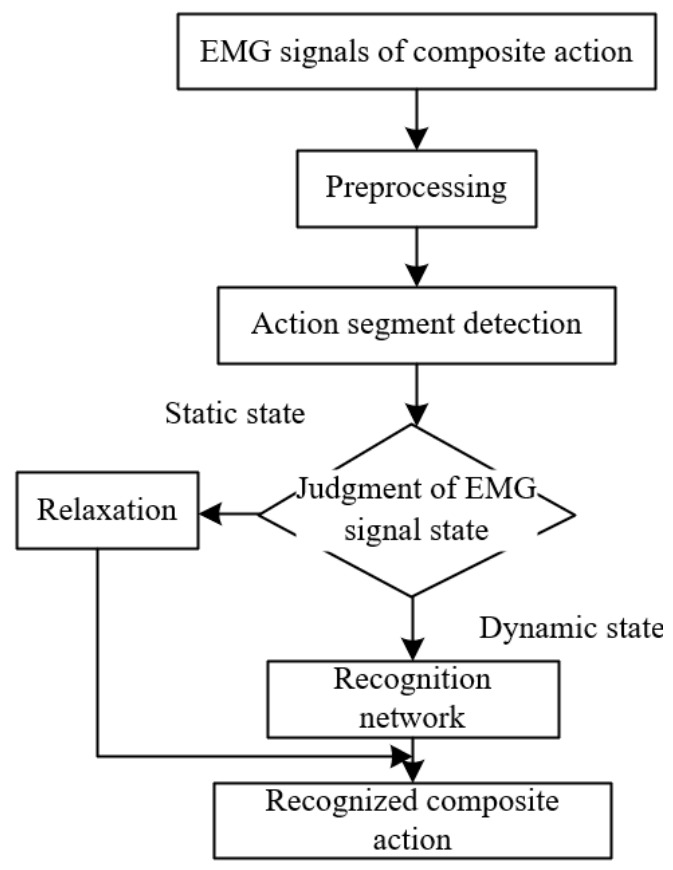

Figure 4. Block diagram of composite action segmentation network

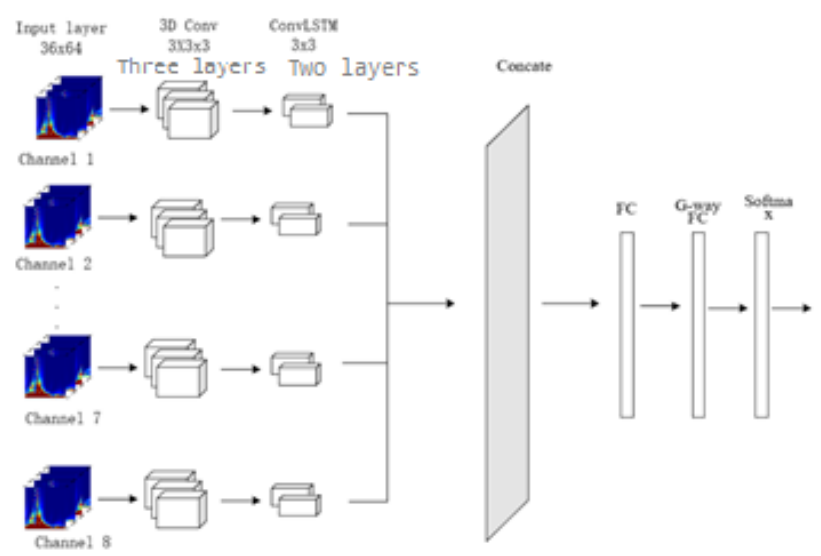

Figure 5. Action recognition network

Table 1. Parameter settings of DCNN

\begin{tabular}{cccc}
\hline Network layer & Kernel size & Filter number & Step length \\
\hline Conv3D1 & $3 \times 3 \times 3$ & 32 & 1 \\
Max Pooling & & & $1 \times 2 \times 2$ \\
Conv3D 2 & $3 \times 3 \times 3$ & 64 & 1 \\
Max Pooling & & & $1 \times 2 \times 2$ \\
Conv3D 3 & $3 \times 3 \times 3$ & 128 & 1 \\
\hline
\end{tabular}

Table 2. Parameter settings of ConvLSTM

\begin{tabular}{cccc}
\hline Network layer & Kernel size & Filter number & Step length \\
\hline ConvLSTM1 & $3 \times 3$ & 128 & 1 \\
ConvLSTM2 & $3 \times 3$ & 64 & 1 \\
ConvLSTM3 & $3 \times 3$ & 128 & 1 \\
\hline
\end{tabular}

\section{EXPERIMENTS}

\subsection{Composite action recognition and results analysis}

The designed action recognition network was trained on every single action dataset. Then, the pretrained network and parameters were further trained on each composite action dataset.

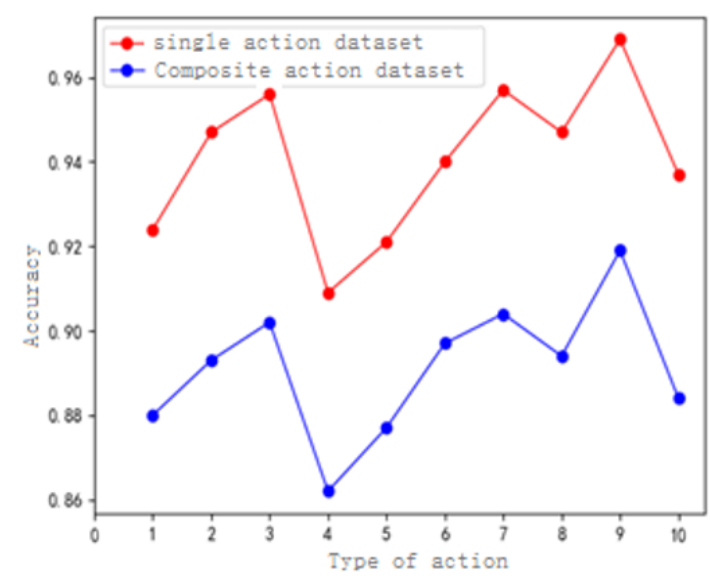

Figure 6. Recognition rates of EMG signals of single and composite actions

Figure 6 shows that the designed network trained by single action dataset achieved very different recognition rates of the ten hand actions from that trained by composite action dataset. The recognition effect on the EMG signals of single action was much better than that on those of composite action. The main reason is as follows.

During the recognition of isolated actions, there are only three states of the signals: static state, dynamic state, and static-dynamic transition state. Besides, the signals contain a single type of information. That is why single actions are easy to detect. However, real-life actions are composed of multiple single actions. The EMG signals of such composite actions carry rich information, and change between four states: static state, dynamic state, static-dynamic transition state, and dynamic-dynamic transition state. The transitions between different actions in the composite action make it hard to fully extract the effective information, adding difficulty to action recognition. Besides, the composite actions take a long time to complete. The resulting muscle fatigue will also suppress the recognition effect.

\subsection{Model optimization}

A major difficulty in the EMG signal recognition of composite actions lies in the differentiation between actions during the dynamic-dynamic transition state. The EMG signals often include noises of this state, making it hard to segment the signals. This paper tries to recognize the EMG signals of composite actions with sliding window, and optimize the recognition result by the voting mechanism.

\subsubsection{Design of sliding window}

There is a maximum delay of $300 \mathrm{~ms}$ from the generation of sEMG signals to the execution of an action. To avoid excessive delay, it is necessary to design a sliding window with a suitable length for EMG signal recognition. In this paper, 200ms-long sliding windows (144 sampling points) is 
designed, and moved across 48 sampling points on the original data. There is a certain overlap between windows. With these windows, the EMG signals of composite actions were divided into multiple partly overlapping segments (Figure 7). Then, the action recognition network was applied to process these segments one after another.

During the sliding of each window, the window was placed at the first sampling point of the EMG signal series, and its window size was defined. After that, the signal series in the window were imported to the deep learning-based action
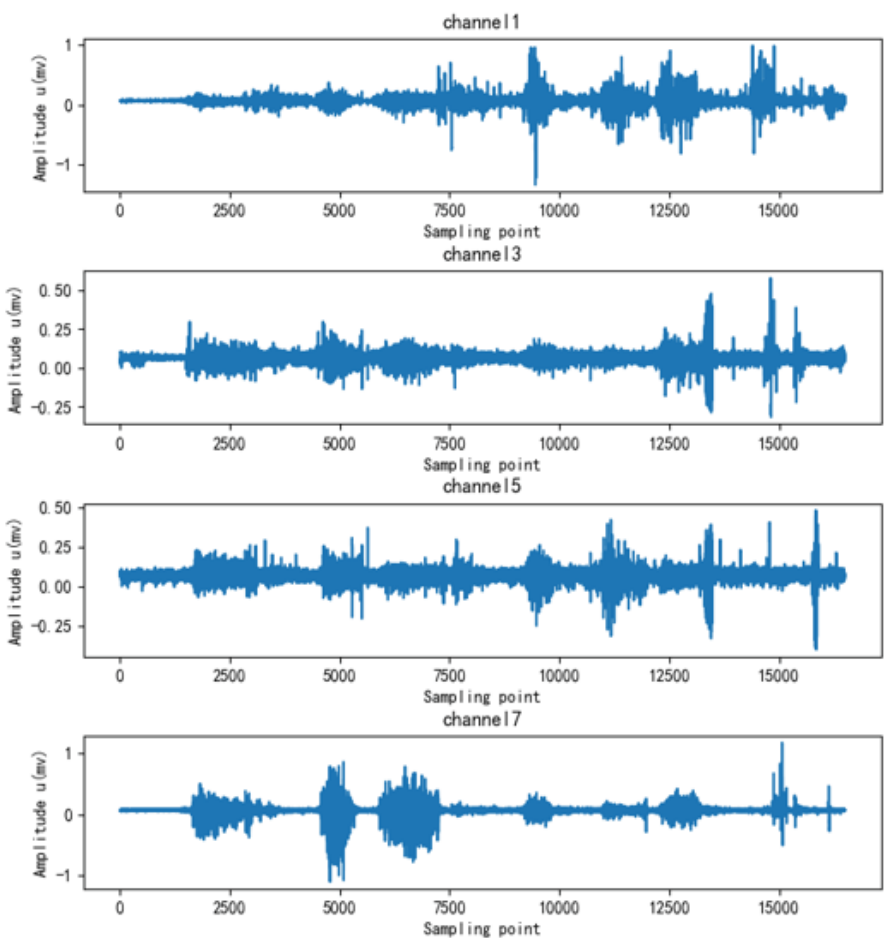

Figure 7. EMG signals of the ten composite actions

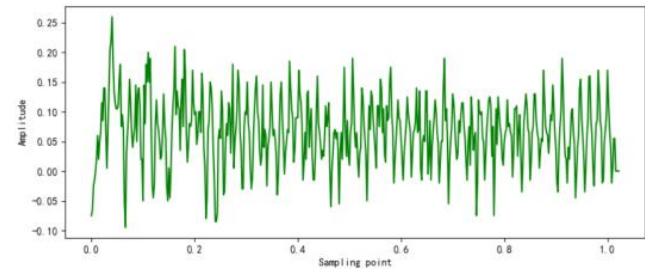

(a) Frame number of the current waveform

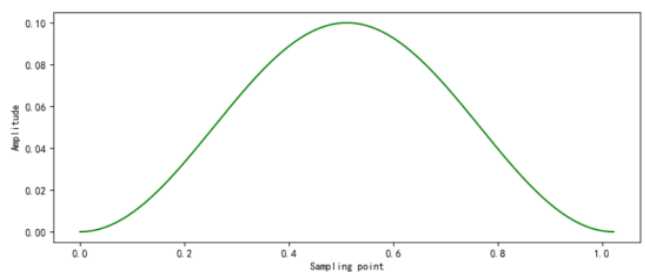

(b) Hamming window

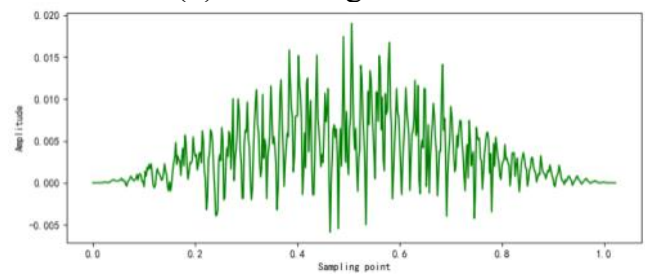

(c) Signal after the application of Hamming window

Figure 8. EMG signals after framing and Hamming window processing recognition network for recognition and classification. Thus, the class of the action in the EMG signal series of the current window could be obtained. Then, the time window was slid along the direction of the action series by the preset step length, producing the class of the action in the second window. Next, the action recognition network was called to complete action recognition and classification. The windows were thus slid in turn across the EMG signal series of the entire composite action. In this way, the action class in each window could be determined for the whole composite action.
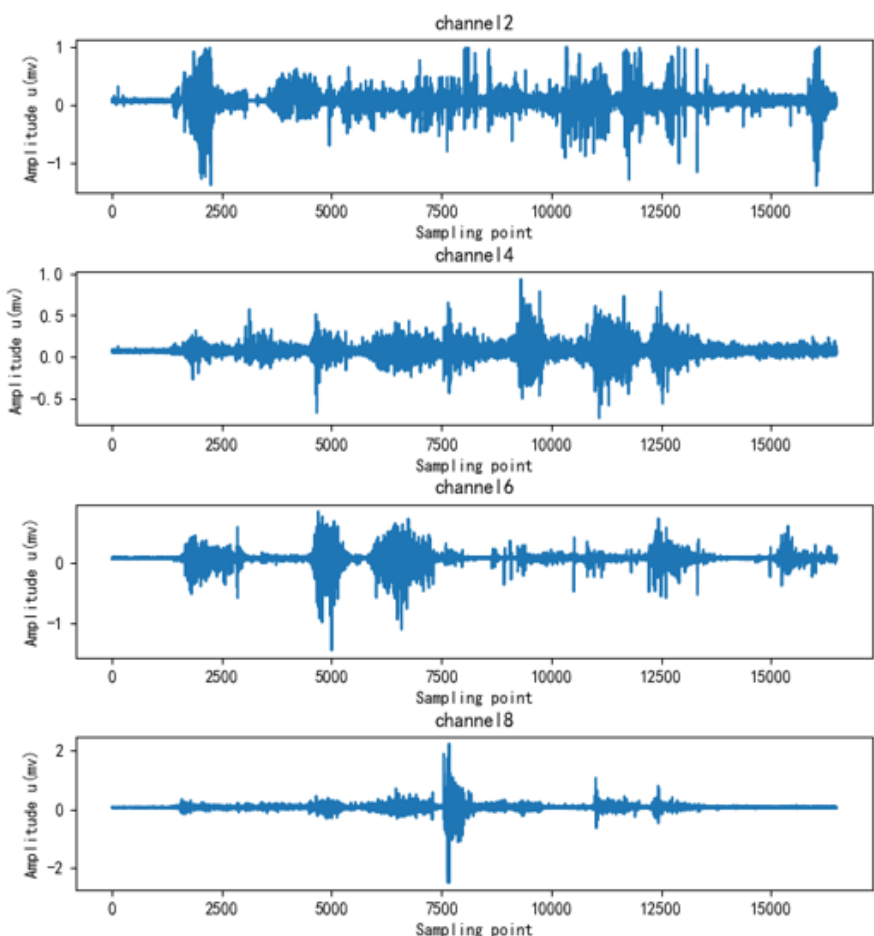

Normally, window is needed for signal truncation and framing. This is because frequency-domain energy inevitably leaks during the truncation; the window function can smooth the discontinuous changes at the truncated point, and suppress noise interference. The Hamming window does better than rectangular window in eliminating high-frequency interference and energy leakage. Its main lobe can be widened and shortened, and the size of its side lobes can be significantly reduced. Let $L$ be the window length. Then, the size of Hamming window $\omega_{n}$ can be obtained as:

$$
\omega_{n}=\left\{\begin{array}{lr}
0.54-0.46 \cos \frac{2 \pi n}{L-1}, & 0 \leq n<L-10 \\
0, & \text { otherwise }
\end{array}\right.
$$

This paper adds the Hamming window to process the EMG signals. Figure 8 provides the Hamming window function, post-framing EMG signals, and post-framing EMG signals processed by Hamming window. It can be seen that the application of Hamming window suppressed the EMG signals on the two sides. To prevent excessive attenuation and maintain signal integrity, a suitable frame shift should be selected for framing.

\subsubsection{Wavelet denoising}

EMG signals, as weak low-frequency signals, are susceptible to interference by the external environment and the 
human body. To ensure recognition accuracy, it is critical to remove the noises from sEMG signals through preprocessing. Therefore, wavelet denoising was implemented in the following steps:

Step 1. Select a suitable wavelet basis to decompose the signal after baseline drift correction, and obtain the low- and high-frequency coefficients of different layers.

Step 2. Determine the threshold for the high-frequency coefficient of each post-decomposition layer through unbiased likelihood estimation, quantify the threshold with the linear attenuation threshold function, and zeroize any coefficient whose absolute value is smaller than the preset threshold, leaving only the high-frequency information.

Step 3. Produce the sEMG signals free of high-frequency noises by reconstructing the low-frequency coefficient of the top layer after wavelet decomposition and the high-frequency information of each layer after thresholding.

After multiple experiments, the wavelet basis function of Sym 10 was selected to decompose the EMG signals into eight layers. The function boasts good local features in time and frequency domains, high smoothness, ease of implementation, and high recognition rate of frequency. Experimental results confirm that the function can effectively remove the noises from sEMG signals. Figure 9 compares the post-baseline drift correction waveform with the denoised waveform. The denoise waveform was much smoother than the sEMG signals after baseline drift correction, yet retained the shape of the original waveform. This means the wavelet analysis method has good filtering effect.

sEMG signals contain many information about muscle actions and muscle fatigue. However, the signals cannot be directly applied, because they are interfered by various noises. This paper proposes a pertinent denoising method for noises like power-line interference, baseline drift, white noise, etc. After noise removal, the sEMG signals are suitable for feature extraction or decomposition. Then, the application value of these signals could crystalize in prosthesis control, gesture recognition, and muscle disease diagnosis and treatment.

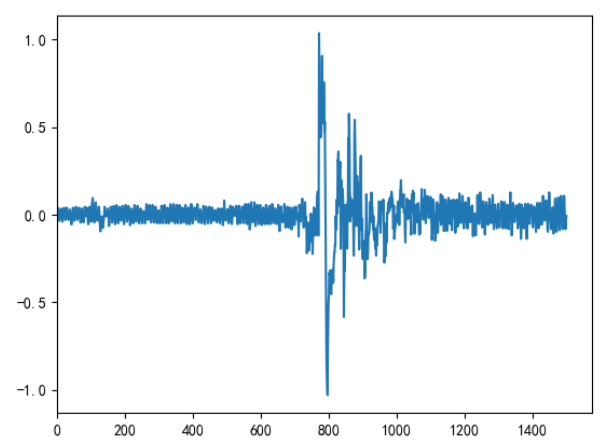

(a) Waveform after baseline drift correction

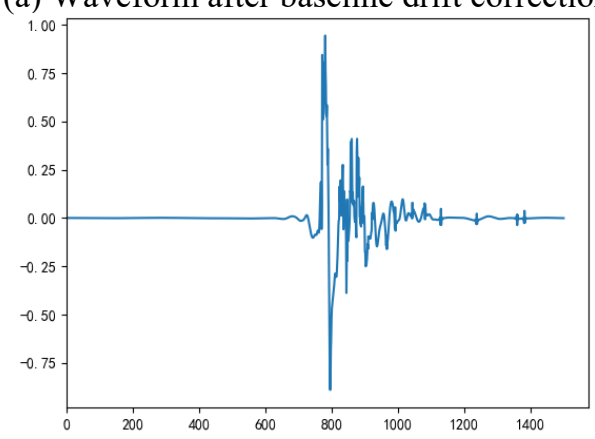

(b) Waveform after wavelet denoising

Figure 9. Waveforms before and after wavelet denoising

\section{EXPERIMENTAL VERIFICATION}

To experimentally verify our method, a simulated driving environment was established on Unity 5.3.1f1. The user information was collected by EMG signal acquisition instrument. Natural interaction with the virtual environment was achieved through gesture recognition. Taking the game scene as the core of training system, the simulation scene was constructed through software modeling. Through EMG signal detection, the patient's instructions were transmitted to the scene, and his/her actions were recognized, laying the basis for rehabilitation training.

\subsection{Definition of gestures and interaction effects}

The composite actions of five subjects were collected offline, preprocessed, and segmented into individual actions. The segmented data were imported to deep learning network for offline training. After the training, the classifier parameters were inputted to the classifier in the host computer software. Then, the experimenter carried out online action recognition on the software, and tested the system performance by the recognition rate. After that, python program was run on the computer, and the trained classifier was used to predict hand actions. Finally, the unmanned vehicle was controlled to complete the corresponding actions.

To better demonstrate the response of the unmanned vehicle to gesture instructions in virtual environment, eight hand actions were defined to control the unmanned vehicle to start, stop, move forward, move backward, turn left, turn right, change to the left lane, and change to the right lane, respectively. To interactively control the vehicle speed, three palm actions were defined to control the vehicle to speed up, slow down, and move at a constant speed, respectively. By these actions, the unmanned vehicle can respond in both direction and speed. The name of each gesture and meaning of each action are summarized in Table 3.

The experiments were prepared the same as the data collection in Section 3.1 Prior to the experiments, every subject was told all the details about the experiments, including method, steps, and matters needing attention. The experiments were kicked off after all subjects expressed the consent.

Table 3. Name of each gesture and meaning of each action

\begin{tabular}{ccc}
\hline $\begin{array}{c}\text { Serial } \\
\text { number }\end{array}$ & Action & Effect \\
\hline 0 & Initial state (static signal) & Constant speed \\
1 & Palm opening & Moving forward \\
2 & Gripping & Moving backward \\
3 & Wrist raising & Speeding up \\
4 & Wrist lowering & Left turn of $90^{\circ}$ \\
5 & Wrist left bending & Left turn of $45^{\circ}$ \\
6 & Wrist right bending & Right turn of $45^{\circ}$ \\
7 & Wrist counterclockwise rotation & Stop \\
8 & Wrist clockwise rotation & Right turn of $90^{\circ}$ \\
10 & Arm raising & Doubling speed \\
11 & Arm lowering & Halving speed \\
\hline
\end{tabular}

\subsection{Action recognition and interaction simulation}

After receiving an instruction, the unmanned vehicle would perform the corresponding action, and record whether the recognition is correct. The program would record the time from the moment the vehicle receives the sEMG signal to the 
moment it completes the action. Each subject need to complete the composite action under each scenario, as designed in Section 3.1 After completing a composite action, he/she could relax his/her arms, and get ready for the next composite action, according to the instructions. A total of ten rounds of experiments were carried out on each subject. Each round should recognize three daily composite actions. There was a $5 \mathrm{~min}$-long break between two adjacent rounds of experiments. Figure 10 shows the photos on the real-time control experiments of the unmanned vehicle.

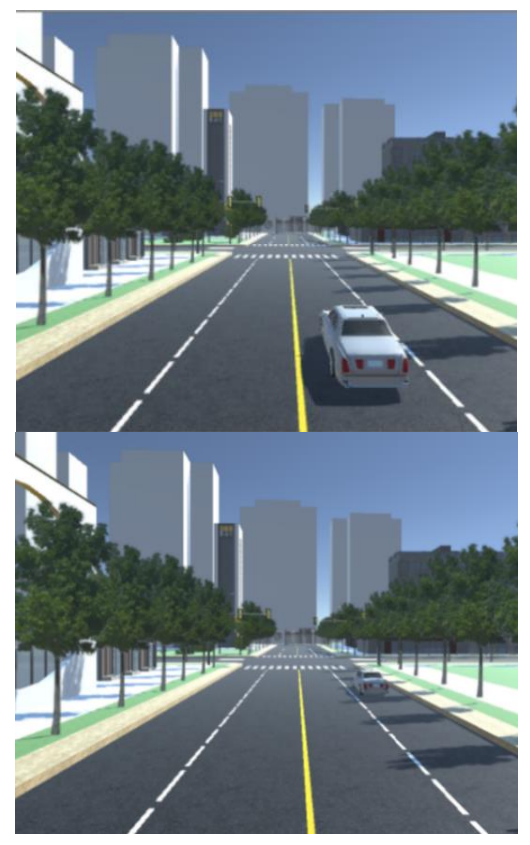

Figure 10. Arm raising plus wrist right bending: the interactive effect of speeding up and changing to the right lane

After all subjects completed ten rounds of experiment, the results of each subject were sorted out. Table 4 present the experimental results on five subjects.

Table 4. Results of interaction experiments

\begin{tabular}{ccc}
\hline $\begin{array}{c}\text { Serial number } \\
\text { of subjects }\end{array}$ & $\begin{array}{c}\text { Recognition rate } \\
\text { before denoising }\end{array}$ & $\begin{array}{c}\text { Recognition rate } \\
\text { after denoising }\end{array}$ \\
\hline 1 & $88.87 \%$ & $90.22 \%$ \\
2 & $89.64 \%$ & $91.35 \%$ \\
3 & $87.13 \%$ & $90.76 \%$ \\
4 & $86.59 \%$ & $89.87 \%$ \\
5 & $89.60 \%$ & $91.28 \%$ \\
Mean & $88.37 \%$ & $90.7 \%$ \\
\hline
\end{tabular}

As shown in Table 4, the five subjects executed the actions with an average accuracy of $90.7 \%$ in the virtual rehabilitation training system. This proves the feasibility of our model in recognizing patient's intention and completing rehabilitation training.

\section{CONCLUSIONS}

To recognize the EMG signals produced from the composite actions of rehabilitation training, this paper reviews the theories on deep learning, and proposes a hybrid algorithm based on 3D CNN and ConvLSTM for action recognition.
Based on the algorithm, an optimal recognition model was developed for composite actions. Finally, action instructions were given in Unity 3D environment, and EMG signals were taken as inputs to control the driving of unmanned vehicle. The results show that our recognition system can recognize the patient's intention in rehabilitation training, encourage $\mathrm{him} /$ her to actively complete the training actions, and improve the training effect. The main contributions are as follows:

(1) To overcome the insufficient extraction of time-domain features from EMG signals, a 3D CNN-Conv LSTM action recognition network was constructed. The network can accurately recognize daily composite actions of hands, and performed well on the EMG signal data of untrained subjects.

(2) The authors created a composite action recognition model, enhanced the ability of feature extraction of EMG signals with sliding window, and optimized the action recognition network by adding the voting mechanism. During the experiments, a self-designed dataset was prepared to test the proposed network. The results show that the optimized model achieved a good recognition rate.

(3) Through virtual simulation experiments, our action recognition system was proved feasible for unmanned vehicle control in virtual environment, using EMG signals.

\section{ACKNOWLEDGEMENT}

This paper was supported by National Fund Youth Project (Grant No.: 11804263).

\section{REFERENCES}

[1] Lin, K., Wu, C., Huang, X., Ding, Q., Gao, X. (2015). A robust gesture recognition algorithm based on surface EMG. 2015 Seventh International Conference on Advanced Computational Intelligence (ICACI), Wuyi, China, pp. 131-136. https://doi.org/10.1109/ICACI.2015.7184763

[2] Pukhova, V., Gorelova, E., Ferrini, G., Burnasheva, S. (2017). Time-frequency representation of signals by wavelet transform. 2017 IEEE Conference of Russian Young Researchers in Electrical and Electronic Engineering (EIConRus), St. Petersburg and Moscow, Russia, pp. https://doi.org/10.1109/EIConRus.2017.7910658

[3] Luo, K., Du, K., Cai, Z., Li, J., Wang, Z., Cuschieri, A. (2017). A modified frequency slice wavelet transform for physiological signal time-frequency analysis. In 2017 Chinese Automation Congress (CAC), Jinan, China, pp. 3441-3444. https://doi.org/10.1109/CAC.2017.8243375

[4] Piao, X., Hu, Y., Gao, J., Sun, Y., Yang, X., Yin, B., Li, G. (2020). Kernel clustering on symmetric positive definite manifolds via double approximated low rank representation. 2020 IEEE International Conference on Multimedia and Expo (ICME), London, UK, pp. 1-6. https://doi.org/10.1109/ICME46284.2020.9102821

[5] Piao, X., Hu, Y., Gao, J., Sun, Y., Yin, B. (2019). Double nuclear norm based low rank representation on Grassmann manifolds for clustering. Proceedings of the IEEE/CVF Conference on Computer Vision and Pattern Recognition, Long Beach, CA, USA, pp. 12075-12084. https://doi.org/10.1109/CVPR.2019.01235 
[6] Feng, J., Wu, X., Chen, J., Zhang, X., Tang, X., Li, D. (2019). Joint multilayer spatial-spectral classification of hyperspectral images based on CNN and ConvLSTM. IGARSS 2019-2019 IEEE International Geoscience and Remote Sensing Symposium, Yokohama, Japan, pp. 588-591.

https://doi.org/10.1109/IGARSS.2019.8897819

[7] Atzori, M., Gijsberts, A., Castellini, C., Caputo, B., Hager, A.G.M., Elsig, S., Müller, H. (2014). Electromyography data for non-invasive naturallycontrolled robotic hand prostheses. Scientific Data, 1(1): 140053. https://doi.org/10.1038/sdata.2014.53

[8] Hu, W.S., Li, H.C., Pan, L., Li, W., Tao, R., Du, Q. (2020). Spatial-spectral feature extraction via deep ConvLSTM neural networks for hyperspectral image classification. IEEE Transactions on Geoscience and Remote Sensing, 58(6): 4237-4250. https://doi.org/10.1109/TGRS.2019.2961947

[9] Chen, W., Zhang, L., Xi, Y., Wang, Y., Li, Y. (2019). Polarimetric HRRP Target Recognition Based on Convlstm. IGARSS 2019-2019 IEEE International Geoscience and Remote Sensing Symposium, Yokohama, Japan, pp. 334-337. https://doi.org/10.1109/IGARSS.2019.8898645

[10] Huang, D., Chen, B. (2019). Surface EMG decoding for hand gestures based on spectrogram and CNN-LSTM. 2019 2nd China Symposium on Cognitive Computing and Hybrid Intelligence (CCHI), Xi'an, China, pp. 123 126. https://doi.org/10.1109/CCHI.2019.8901936

[11] Wang, B., Hu, Y., Gao, J., Sun, Y., Ju, F., Yin, B. (2020). Learning adaptive neighborhood graph on Grassmann manifolds for video/image-set subspace clustering. IEEE Transactions on Multimedia, 23: 216-227. https://doi.org/10.1109/TMM.2020.2975394

[12] Wang, B., Hu, Y., Gao, J., Ali, M., Tien, D., Sun, Y., Yin, B. (2018). Low rank representation on SPD matrices with log-Euclidean metric. Pattern Recognition, 76: 623-634. https://doi.org/10.1016/j.patcog.2017.07.009

[13] Davydova, N., Davydov, M., Osipov, A., Mezhennaya, M. (2019). Complex analysis of human movements based on the identification of amplitude-time characteristics of electromyographic patterns. Global Journal of Research in Engineering, 19(5): 18-26.

[14] Luo, R., Sun, S., Zhang, X., Tang, Z., Wang, W. (2019). A low-cost end-to-end sEMG-based gait sub-phase recognition system. IEEE Transactions on Neural Systems and Rehabilitation Engineering, 28(1): 267-276. https://doi.org/10.1109/TNSRE.2019.2950096

[15] Dimyan, M.A., Cohen, L.G. (2011). Neuroplasticity in the context of motor rehabilitation after stroke. Nature Reviews Neurology, 7(2): 76-85. https://doi.org/10.1038/nrneurol.2010.200

[16] Kim, T., Kim, S., Lee, B. (2016). Effects of action observational training plus brain - computer interface based functional electrical stimulation on paretic arm motor recovery in patient with stroke: a randomized controlled trial. Occupational Therapy International, 23(1): 39-47. https://doi.org/10.1002/oti.1403

[17] Amsüss, S., Goebel, P.M., Jiang, N., Graimann, B., Paredes, L., Farina, D. (2013). Self-correcting pattern recognition system of surface EMG signals for upper limb prosthesis control. IEEE Transactions on Biomedical Engineering, 61(4): 1167-1176. https://doi.org/10.1109/TBME.2013.2296274

[18] Milosevic, B., Benatti, S., Farella, E. (2017). Design challenges for wearable EMG applications. In Design, Automation \& Test in Europe Conference \& Exhibition (DATE), Lausanne, Switzerland, pp. 1432-1437. https://doi.org/10.23919/DATE.2017.7927217

[19] Tian, L., Zimmerman, B., Akhtar, A., Yu, K.J., Moore, M., Wu, J., Rogers, J.A. (2019). Large-area MRIcompatible epidermal electronic interfaces for prosthetic control and cognitive monitoring. Nature Biomedical Engineering, 3(3): 194-205. https://doi.org/10.1038/s41551-019-0347-x

[20] Du, Y., Jin, W., Wei, W., Hu, Y., Geng, W. (2017). Surface EMG-based inter-session gesture recognition enhanced by deep domain adaptation. Sensors, 17(3): 458. https://doi.org/10.3390/s17030458

[21] Quivira, F., Koike-Akino, T., Wang, Y., Erdogmus, D. (2018). Translating sEMG signals to continuous hand poses using recurrent neural networks. In 2018 IEEE EMBS International Conference on Biomedical \& Health Informatics (BHI), Las Vegas, NV, USA, pp. 166-169. https://doi.org/10.1109/BHI.2018.8333395

[22] Guo, S., Pang, M., Gao, B., Hirata, H., Ishihara, H. (2015). Comparison of sEMG-based feature extraction and motion classification methods for upper-limb movement. Sensors, 15(4): 9022-9038. https://doi.org/10.3390/s150409022

[23] Du, M., Wang, Z., Xu, Y., Bao, G., Guo, F., Yang Q., Zhang, L. (2018). Hand action pattern recognition based on multi-channel sEMG wavelet packet decomposition feature. Computer Measurement \& Control, 6(26): 160163. https://doi.org/10.16526/j.cnki.114762/tp.2018.06.041

[24] Luo, T., Yan, T. (2008). Classification of surface electromyography patterns by using HMM. Journal of Huazhong University of Science and Technology (Nature Science Edition), 36(4): 72-75. https://doi.org/10.3321/j.issn:1671-4512.2008.04.020

[25] Ma, D., Yang, Z., Wang, L. (2020). Research on SEMG signal denoising based on two improved threshold functions. Modern Electronics Technique, 43(1): 67-71, 75. $373 x .2020 .01 .018$ https://doi.org/10.16652/j.issn.1004-

[26] Atzori, M., Cognolato, M., Müller, H. (2016). Deep learning with convolutional neural networks applied to electromyography data: A resource for the classification of movements for prosthetic hands. Frontiers in Neurorobotics, 10 : https://doi.org/10.3389/fnbot.2016.00009

[27] Hochreiter, S., Schmidhuber, J. (1997). Long short-term memory. Neural Computation, 9(8): 1735-1780.

[28] Côté-Allard, U., Fall, C.L., Drouin, A., CampeauLecours, A., Gosselin, C., Glette, K., Gosselin, B. (2019). Deep learning for electromyographic hand gesture signal classification using transfer learning. IEEE Transactions on Neural Systems and Rehabilitation Engineering, 27(4) 760-771. https://doi.org/10.1109/TNSRE.2019.2896269 